\title{
Correction to: Symmetry-Aware Face Completion with Generative Adversarial Networks
}

\author{
Jiawan Zhang, Rui Zhan, Di Sun, and Gang Pan
}

\section{Correction to:}

Chapter "Symmetry-Aware Face Completion with Generative Adversarial Networks" in: C. V. Jawahar et al. (Eds.): Computer Vision - ACCV 2018, LNCS 11364, https://doi.org/10.1007/978-3-030-20870-7_18

In the original version of this book the address of the author Di Sun was incorrect. This has now been corrected. 